\title{
Short-Time Heat Content Asymptotics via the Wave and Eikonal Equations
}

\author{
Nathanael Schilling ${ }^{1}$
}

Received: 23 January 2020 / Published online: 12 May 2020

(c) The Author(s) 2020

\section{Abstract}

In this short paper, we derive an alternative proof for some known (van den Berg \& Gilkey 2015) short-time asymptotics of the heat content in a compact full-dimensional submanifolds $S$ with smooth boundary. This includes formulae like

$$
\int_{S} \exp (t \Delta)\left(f \mathbb{1}_{S}\right) \mathrm{d} V=\int_{S} f \mathrm{~d} V-\sqrt{\frac{t}{\pi}} \int_{\partial S} f \mathrm{~d} A+o(\sqrt{t}), \quad t \rightarrow 0^{+},
$$

and explicit expressions for similar expansions involving other powers of $\sqrt{t}$. By the same method, we also obtain short-time asymptotics of $\int_{S} \exp \left(t^{m} \Delta^{m}\right)\left(f \mathbb{1}_{S}\right) \mathrm{d} V$, $m \in \mathbb{N}$, and more generally for one-parameter families of operators $t \mapsto k(\sqrt{-t \Delta})$ defined by an even Schwartz function $k$.

Keywords Heat equation · Heat content · Riemannian manifolds · Geometrical optics

\section{Introduction}

Let $(M, g)$ be a complete, boundaryless, ${ }^{1}$ oriented Riemannian manifold with Laplace-Beltrami operator $\Delta$, and volume $\mathrm{d} V$. On a codimension-1 submanifold of $M$, we write $\mathrm{d} A$ for the induced surface (hyper)-area form. The heat semi-group $T_{t}:=\exp (t \Delta)$ acting on $L^{2}(M, \mathrm{~d} V)$ is well defined ( $\Delta$ is essentially self-adjoint on $C_{c}^{\infty}(M)$ [2]) and its behaviour as $t \rightarrow 0^{+}$has been extensively investigated in the literature. Specifically, for a set $S \subset M$, the heat content of the form

\footnotetext{
${ }^{1}$ We assume that $M$ has no boundary for the sake of simplicity, and the method presented here can be adapted to more general manifolds with boundary provided that $S$ is compactly contained in the interior of $M$. If this is not the case, such as in the classical heat content setting as in [13], it should be possible to obtain similar results by modifying the geometrical optics construction used.
}

\footnotetext{
$\bowtie \quad$ Nathanael Schilling schillna@ma.tum.de

1 Zentrum Mathematik, Technische Universität München, Boltzmannstr. 3, 85748 Garching bei München, Germany
} 
$\Omega_{S, f}(t):=\int_{S} T_{t}\left(f \mathbb{1}_{S}\right) \mathrm{d} V, f \in C^{\infty}(M)$, has recently received much attention; see, for instance, $[7,11,12]$ and the references therein.

Let us briefly recall some known results. On $\mathbb{R}^{n}$, sets $S$ of finite perimeter $P(S)$ are characterized by [7, Thm. 3.3 ]

$$
\lim _{t \rightarrow 0^{+}} \sqrt{\frac{\pi}{t}}\left(\Omega_{S, \mathbb{1}_{M}}(0)-\Omega_{S, \mathbb{1}_{M}}(t)\right)=P(S) .
$$

Extensions of this idea to abstract metric spaces are given in [6]. In the setting of compact manifolds $M$ (or $M=\mathbb{R}^{n}$ ) and $S$ a full-dimensional submanifold with smooth boundary $\partial S$, the authors of [12] show that

$$
\Omega_{S, f}(t)=\sum_{j=0}^{\infty} \beta_{j} t^{\frac{j}{2}}, \quad t \rightarrow 0^{+}
$$

where the coefficients $\beta_{j}$ depend on $S, f$ and the geometry of $M$. The setting of [12] is more general, amongst other things it includes $f$ which have singularities. Some of the coefficients obtained in [12, corollary 1.7] are

$$
\beta_{0}=\int_{S} f \mathrm{~d} V, \quad \beta_{1}=-\frac{1}{\sqrt{\pi}} \int_{\partial S} f \mathrm{~d} A, \quad \beta_{2}=\frac{1}{2} \int_{S} \Delta f \mathrm{~d} V .
$$

Extensions to some non-compact manifolds $M$ and certain non-compact $S$ are in [11].

Both Eqs. (1) and (2) are proven with significant technical effort, yielding strong results. For example, in [7], explicit knowledge of the fundamental solution of the heat equation is used to obtain Eq. (1) for $C^{1,1}$-smooth $\partial S$, after which geometric measure theory is used. Similarly, [12] requires pseudo-differential calculus and invariance theory.

Our aim is to show that slightly weaker results can be obtained by considerably lower technical effort. In contrast to [7], we treat only compact $S$ with smooth boundary, and do not allow $f$ to have singularities like [12] does. On the other hand, we put no further restrictions than completeness on $M$. The proof presented here is simple, comparatively short, and provides an alternative differential geometric/functional analytic point of view to questions regarding heat content. Moreover, this approach is readily extended to some other PDEs including the semi-group generated by $\Delta^{m}$. Observe that $T(t)=k(\sqrt{-t \Delta})$ with $k(x)=\exp \left(-x^{2}\right)$. We allow $k$ to be an arbitrary even Schwarz function, with $\Omega_{S, f}(t)=\int_{S} k(\sqrt{-t \Delta})\left(f \mathbb{1}_{S}\right) \mathrm{d} V$ and will prove:

Theorem 1 Let $M$ be a complete Riemannian manifold with Laplace-Beltrami operator $\Delta$, Riemannian volume $\mathrm{d} V$ and induced (hyper) area form $\mathrm{d} A$. Let $S \subset M$ be a compact full-dimensional submanifold with smooth boundary. For $f \in C^{\infty}(M)$ and $N \in \mathbb{N}$

$$
\Omega_{S, f}(t)=\sum_{j=0}^{N} \beta_{j} t^{\frac{j}{2}}+o\left(t^{\frac{N}{2}}\right), \quad t \rightarrow 0^{+}
$$


for constants $\left(\beta_{j}\right)_{j=0}^{N}$ described further in the next theorem.

With the $j$ th derivative $k^{(j)}$ (for $j \in \mathbb{N}_{0}$ ), let $r_{j}:=(-1)^{j / 2} k^{(j)}(0)$ for $j$ even and $r_{j}:=(-1)^{(j-1) / 2} \int_{0}^{\infty} \frac{2 k^{j}(s)}{-\pi s} \mathrm{~d} s$ for $j$ odd. Let $\varphi$ locally be the signed distance function (see also [8, Sect. 3.2.2]) to $\partial S$ with $S=\varphi^{-1}([0, \infty)$ ), and denote by $\nabla$ and - the gradient and (metric) inner product, respectively. The vector field $v:=-\nabla \varphi$ is outer unit normal at $\partial S$.

Theorem 2 The coefficients of Theorem 1 satisfy $\beta_{0}=r_{0} \int_{S} f \mathrm{~d} V$ and $\beta_{1}=$ $-\frac{1}{2} r_{1} \int_{\partial S} f \mathrm{~d} A$. For even $j \in \mathbb{N}_{\geq 2}$,

$$
\beta_{j}=\frac{r_{j}}{j !} \int_{S} \frac{1}{2} \Delta^{j / 2} f \mathrm{~d} V .
$$

Moreover, given the Lie-derivative $\mathcal{L}_{v}$ with respect to $v$,

$$
\beta_{3}=\frac{r_{3}}{2 \cdot 3 !} \int_{\partial S} \mathcal{L}_{v}\left(-\mathcal{L}_{v}+\frac{1}{2} \Delta \varphi\right) f-\frac{1}{2} \Delta f+\frac{1}{2}\left(-\mathcal{L}_{v}+\frac{1}{2} \Delta \varphi\right)^{2} f \mathrm{~d} A
$$

similar expression can be found also for larger odd values of $j$ (see Sect. 3).

The properties of the signed distance function $\varphi$ may be used to express terms appearing in Theorem 2 using other quantities. For example, its Hessian $\nabla^{2} \varphi$ is the second fundamental form on the tangent space of $\partial S$ [3, Chap. 3], and thus $\frac{1}{2} \Delta \varphi$ is the mean curvature.

Our approach to prove Theorems 1 and 2 is to combine 3 well-known facts:

(A) The short-time behaviour of the heat flow is related to the short-time behaviour of the wave equation (cf. [1]).

(B) The short-time behaviour of the wave equation with discontinuous initial data is related to the short-time behaviour of the eikonal equation (cf. 'geometrical optics' and the progressing wave expansion [10]).

(C) The short-time behaviour of the wave and eikonal equations with initial data $f \mathbb{1}_{S}$ is directly related to the geometry of $M$ near $\partial S$.

Though points (A)-(C) are well known in the literature, they have (to the best of our knowledge) not been applied to the study of heat content so far.

A significant portion of $(C)$ will rest on an application of the Reynolds transport theorem. Here, denote by $\Phi^{s}$ the time-s flow of the vector field $v=-\nabla \varphi$. For small $s$, the (half) tubular neighbourhood

$$
S^{-s}:=\{x \in M \backslash S: \operatorname{dist}(x, \partial S) \leq s\}
$$

satisfies $S \cup S^{-s}=\Phi^{s}(S)$. For $a \in C^{\infty}((-\varepsilon, \varepsilon) \times M)$, by [5, Chap. V, Prop. 5.2],

$$
\begin{aligned}
\left.\frac{\mathrm{d}}{\mathrm{d} s} \int_{S^{-s}} a(s, \cdot) \mathrm{d} V\right|_{s=0} & =\left.\frac{\mathrm{d}}{\mathrm{d} s}\left(\int_{S^{-s} \cup S} a(s, \cdot) \mathrm{d} V-\int_{S} a(s, \cdot) \mathrm{d} V\right)\right|_{s=0} \\
& =\int_{S} \mathcal{L}_{\tilde{v}}[a(0, \cdot) \mathrm{d} V]=\int_{\partial S} a(0, \cdot) \mathrm{d} A
\end{aligned}
$$


The last equation is a consequence of Cartan's magic formula and Stokes' theorem, where we use that $\mathrm{d} V(v, \cdot)=\mathrm{d} A(\cdot)$ on $\partial S$.

\section{Proof for $\beta_{0}, \beta_{1}$}

By Fourier theory (for non-Gaussian $k$, the formulae must be adapted),

$$
k(t)=\exp \left(-t^{2}\right)=\int_{0}^{\infty} \hat{k}(s) \cos (t s) \mathrm{d} s \text { with } \hat{k}(s):=\frac{1}{\sqrt{\pi}} \exp \left(\frac{-s^{2}}{4}\right) .
$$

On the operator level, this yields the well-known formula [10, Sect. 6.2]

$$
T_{t}=\exp (t \Delta)=\int_{0}^{\infty} \hat{k}(s) \cos (s \sqrt{-t \Delta}) \mathrm{d} s .
$$

The operator $W^{s}:=\cos (s \sqrt{-\Delta})$ is the time- $s$ solution operator for the wave equation with zero initial velocity, in particular $u(s, x):=\left(W^{s} f \mathbb{1}_{S}\right)(x)$ (weakly) satisfies $\left(\partial_{t}^{2}-\Delta\right) u=0$. Let $\langle\cdot, \cdot\rangle$ denote the $L^{2}(M, \mathrm{~d} V)$ inner product. Using Eq. (5),

$$
\left\langle T_{t} f \mathbb{1}_{S}, \mathbb{1}_{S}\right\rangle=\int_{0}^{\infty} \hat{k}(s)\left\langle W_{s \sqrt{t}} f \mathbb{1}_{S}, \mathbb{1}_{S}\right\rangle \mathrm{d} s .
$$

Similar reasoning has been used to great effect in [1] to derive heat-kernel bounds by making use of the finite propagation speed of the wave equation. As in [1], finite propagation speed yields for $s \geq 0$ that $\left\langle W_{s} f \mathbb{1}_{S}, \mathbb{1}_{M \backslash S\rangle}\right\rangle\left\langle W_{s} f \mathbb{1}_{S^{s}}, \mathbb{1}_{S^{-s}}\right\rangle$, where $S^{s}:=(M \backslash S)^{-s}$ is defined like Eq. (3). Even if $\mathbb{1}_{M \backslash S} \notin L^{2}(M, \mathrm{~d} V)$, we have just seen that the inner product $\left\langle W_{S} f \mathbb{1}_{S}, \mathbb{1}_{M \backslash S}\right\rangle$ is nevertheless well defined. In [1], it is further observed that $\left\|W_{s}\right\| \leq 1$. Using the Cauchy-Schwarz inequality and assuming $f=\mathbb{1}_{M}$, Eq. (4) yields

$$
h(s):=\left\langle W_{s} f \mathbb{1}_{S^{s}}, \mathbb{1}_{S^{-s}}\right\rangle \leq\left\|\mathbb{1}_{S^{s}}\right\|_{2}\left\|\mathbb{1}_{S^{-s}}\right\|_{2} \leq s \int_{\partial S} \mathrm{~d} A+o(s), \quad s \rightarrow 0^{+} .
$$

In addition, $\left|\left\langle W_{S} f \mathbb{1}_{S}, \mathbb{1}_{S}\right\rangle\right| \leq\left\|f \mathbb{1}_{S}\right\|_{2}\left\|\mathbb{1}_{S}\right\|_{2}$ for all $s \geq 0$, in particular as $s \rightarrow \infty$. We conclude with some calculations (cf. Lemma 3), that

$$
\begin{aligned}
\left\langle T_{t} \mathbb{1}_{S}, \mathbb{1}_{S}\right\rangle & =\int_{0}^{\infty} \hat{k}(s)\left(\left\langle W_{s \sqrt{t}} \mathbb{1}_{S}, \mathbb{1}_{M}\right\rangle-\left\langle W_{s \sqrt{t}} \mathbb{1}_{S}, \mathbb{1}_{M \backslash S}\right\rangle\right) \mathrm{d} s \\
& =\left\langle\mathbb{1}_{S}, \mathbb{1}_{M}\right\rangle-\int_{0}^{\infty} \hat{k}(s) h(s \sqrt{t}) \mathrm{d} s \\
& \geq \int_{S} \mathrm{~d} V-2 \sqrt{\frac{t}{\pi}} \int_{\partial S} \mathrm{~d} A+o(\sqrt{t}), \quad t \rightarrow 0^{+} .
\end{aligned}
$$

This is weaker than the desired estimate, and restricts to $f=\mathbb{1}_{M}$. The problem is that the estimates in Eq. (6) are too crude. To improve them, we instead approximate 
the solution $u$ to the wave equation with geometrical optics, using the "progressing wave" construction described in [10, Sect. 6.6], some details of which we recall here. The basic idea is that $u$ is in general discontinuous, with an outward — and an inwardmoving discontinuity given by the zero level-set of functions $\varphi^{+}$and $\varphi^{-}$, respectively. The functions $\varphi^{ \pm}$satisfy the eikonal equation $\partial_{t} \varphi= \pm\left|\nabla \varphi^{ \pm}\right|$with initial value $\varphi^{ \pm}(0, \cdot)=\varphi(\cdot)$. Equivalently, using the (nonlinear) operator $E w:=\left(\partial_{t} w\right)^{2}-|\nabla w|^{2}$, the functions $\varphi^{ \pm}$satisfy $E\left(\varphi^{ \pm}\right)=0$. Our analysis is greatly simplified by choosing the initial $\varphi$ to (locally) be the signed distance function to $\partial S$. The eikonal equation is then $\partial_{t} \varphi^{ \pm}= \pm|\nabla \varphi|= \pm|-v|= \pm 1$, i.e. $\varphi^{ \pm}(x, t)=\varphi(x) \pm t$.

The progressing wave construction further makes use of two (locally existing and smooth) solutions $a_{0}^{ \pm}$to the first-order transport equations $\pm \partial_{t} a_{0}^{ \pm}(t, \cdot)+v$. $\nabla a_{0}^{ \pm}(t, x)=\frac{1}{2} a_{0}^{ \pm} \Delta \varphi^{ \pm}$. Observe that with the Heaviside function $\theta: \mathbb{R} \rightarrow \mathbb{R}$, and $\square:=\partial_{t}^{2}-\Delta$, the expression $\square\left(a_{0}^{ \pm} \theta\left(\varphi^{ \pm}\right)\right)$is given by

$$
\left(\theta^{\prime \prime}\left(\varphi^{ \pm}\right) E \varphi^{ \pm}+\square \varphi^{ \pm} \theta^{\prime}\left(\varphi^{ \pm}\right)\right) a_{0}^{ \pm}+2\left(\partial_{t} a_{0}^{ \pm} \partial_{t} \varphi^{ \pm}-\nabla a_{0}^{ \pm} \cdot \nabla \varphi^{ \pm}\right) \theta^{\prime}\left(\varphi^{ \pm}\right)+\square a_{0}^{ \pm} \theta\left(\varphi^{ \pm}\right)
$$

The functions $\varphi^{ \pm}$and $a_{0}^{ \pm}$have been chosen so the above simplifies to

$$
\begin{aligned}
\square\left(a_{0}^{ \pm} \theta\left(\varphi^{ \pm}\right)\right) & =2\left( \pm \partial_{t} a_{0}^{ \pm}+\nabla a_{0}^{ \pm} \cdot v-\frac{1}{2} \Delta \varphi a_{0}^{ \pm}\right) \theta^{\prime}\left(\varphi^{ \pm}\right)+\square a_{0}^{ \pm} \theta\left(\varphi^{ \pm}\right) \\
& =\square a_{0}^{ \pm} \theta\left(\varphi^{ \pm}\right) .
\end{aligned}
$$

Thus $\square\left(a_{0}^{ \pm} \theta\left(\varphi^{ \pm}\right)\right)$is as smooth as $\theta$ is. We use

$$
\tilde{u}(t, x):=a_{0}^{+}(t, x) \theta\left(\varphi^{+}(t, x)\right)+a_{0}^{-}(t, x) \theta\left(\varphi^{-}(t, x)\right)
$$

as an approximation to the discontinuity of the solution $u$ to the wave equation. To maintain consistency with the initial values of $u$, the initial values of the approximation $\tilde{u}$ are chosen to coincide with those of $u$ at $t=0$, this is achieved by setting $a_{0}^{ \pm}(0, \cdot)=$ $\frac{1}{2} f$ so that (at least formally) $\partial_{t} \tilde{u}(0, \cdot)=0$ and also $\tilde{u}(0, \cdot)=\mathbb{1}_{S} f$.

The function $\tilde{u}$ approximates the discontinuous solution $u$ of the wave equation well enough that the function $(s, x) \mapsto u(s, x)-\tilde{u}(s, x)$ is continuous on $[-T, T] \times M$, see $[10$, Sect. 6.6, eq. 6.35]. By construction, $\tilde{u}(0, \cdot)=u(0, \cdot)$. Hence $\mid(u(s, x)-$ $\tilde{u}(s, x) \mid=o(1)$ as $s \rightarrow 0^{+}$, which implies

$$
\left|\left\langle u(s, \cdot), \mathbb{1}_{S^{-s}}\right\rangle-\left\langle\tilde{u}(s, \cdot), \mathbb{1}_{S^{-s}}\right\rangle\right|=o(s) \quad s \rightarrow 0^{+}
$$

As $\nabla \varphi=-v$, for sufficiently small $t$ the sets $\left\{x \in M: \varphi^{+}(t, x)=0\right\}$ (resp. $\left\{x: \varphi^{-}(t, x)=0\right\}$ ) are level sets of $\varphi$ on the outside (resp. inside) of $S$ (see also [10, Sect. 6.6]). By construction, $\theta\left(\varphi^{-}\right)$vanishes outside of $S$ for $t>0$. Consequently, 
using Eq. (4), we see that as $s \rightarrow 0^{+}$,

$$
\begin{aligned}
\left\langle\tilde{u}(s, \cdot), \mathbb{1}_{S^{-s}}\right\rangle & =\int_{S^{-s}} a_{0}^{+}(s, x) \mathbb{1}_{\left\{\varphi^{+}(s, \cdot) \geq 0\right\}}+a_{0}^{-}(s, x) \mathbb{1}_{\left\{\varphi^{-}(s, x) \geq 0\right\}} \mathrm{d} V(x) \\
& =s \int_{\partial S} a_{0}^{+}(0, x) \mathrm{d} A(x)+o(s)=\frac{s}{2} \int_{\partial S} f \mathrm{~d} A+o(s) .
\end{aligned}
$$

Combining Eqs. (9) and (10),

$$
h(s)=\left\langle W_{s} f \mathbb{1}_{S}, \mathbb{1}_{S^{-s}}\right\rangle=\left\langle u(s, \cdot), \mathbb{1}_{S^{-s}}\right\rangle=\frac{s}{2} \int_{\partial S} f \mathrm{~d} A+o(s), \quad s \rightarrow 0^{+} .
$$

Calculations along the lines of Lemma 3 and Eq. (7) yield

$$
\left\langle T_{t} f \mathbb{1}_{S}, \mathbb{1}_{S}\right\rangle=\int_{S} f \mathrm{~d} V-\sqrt{\frac{t}{\pi}} \int_{\partial S} f \mathrm{~d} A+o(\sqrt{t}), \quad t \rightarrow 0^{+},
$$

as claimed.

Lemma 3 Let $j \in \mathbb{N}$ and $\gamma: \mathbb{R}_{\geq 0} \rightarrow \mathbb{R}$. Let $\gamma(s)=s^{j}+o\left(s^{j}\right)$ for $s \rightarrow 0$ and $\gamma(s)=O(1)$ for $s \rightarrow \infty$. Then for $t \rightarrow 0^{+}$,

$$
\int_{0}^{\infty} \gamma(s \sqrt{t}) \hat{k}(s) \mathrm{d} s=t^{\frac{j}{2}}\left\{\begin{array}{ll}
(-1)^{\frac{j}{2}} k^{(j)}(0) & j \text { even } \\
(-1)^{\frac{j-1}{2}} \int_{0}^{\infty} \frac{2 k^{(j)}(s)}{-\pi s} \mathrm{~d} s & j \text { odd }
\end{array}+o\left(t^{\frac{j}{2}}\right) .\right.
$$

With $k(s)=\exp \left(-s^{2}\right)$ and $h(s)=c_{0}+c_{1} s+c_{2} s^{2}+o\left(s^{2}\right)$, this implies

$$
\int_{0}^{\infty} h(s \sqrt{t}) \hat{k}(s) \mathrm{d} s=c_{0}+\frac{2 c_{1}}{\sqrt{\pi}} \sqrt{t}+2 c_{2} t+o(t) .
$$

Proof For even $j$, we obtain Eq. (11) by the Fourier-transform formula for $j$ th derivatives. If $j$ is odd, we also need to multiply by the sign function in frequency space, and then use that the inverse Fourier-transform (unnormalized) of the sign function is given by the principal value p.v. $\left(\frac{2 i}{x}\right)$ [10, Sect. 4], see also [9, Chap. 7]. Equation 11 holds more generally, e.g. if $k$ is an even Schwarz function. Equation 12 may also be verified directly without Eq. (11).

\section{Proof for $\beta_{2}, \beta_{3}, \ldots$}

We now turn to calculating $\beta_{j}$ for $j \geq 2$. We use the $N$ th order progressing wave construction with sufficiently large $N \gg j$. For the sake of simplicity, we write $O\left(t^{\infty}\right)$ for quantities that can be made $O\left(t^{k}\right)$ for any $k \in \mathbb{N}$ by choosing sufficiently large $N$. As in the previous section, the construction is from [10, Sect. 6.6]. With 
$\theta_{0}:=\theta$, and $\theta_{i}(t):=\int_{-\infty}^{t} \theta_{i-1}(s) \mathrm{d} s$ we write

$$
\tilde{u}^{ \pm}(t, x):=\sum_{i=0}^{N} a_{i}^{ \pm}(t, x) \theta_{i}\left(\varphi^{ \pm}(t, x)\right)
$$

Here the functions $a_{0}^{ \pm}$are defined as before, and for $i \geq 1$ the $i$ th order transport equations $\pm \partial_{t} a_{i}^{ \pm}=-v \cdot \nabla a_{i}^{ \pm}+\frac{1}{2} a_{i}^{ \pm} \Delta \varphi^{ \pm}-\frac{1}{2} \square a_{i-1}^{ \pm}$define $a_{i}^{ \pm}$together with initial data $a_{i}^{ \pm}(0, \cdot)=-\frac{1}{2}\left(\partial_{t} a_{i-1}^{+}(0, \cdot)+\partial_{t} a_{i-1}^{-}(0, \cdot)\right)$. As in Eq. (8), one may verify that $\square \tilde{u}^{ \pm}=\square a_{i} \theta_{N}\left(\varphi^{ \pm}\right)$. Writing $\tilde{u}=\tilde{u}^{+}+\tilde{u}^{-}$and

$$
u(t, x)=\tilde{u}^{+}(t, x)+\tilde{u}^{-}(t, x)+R_{N}(t, x),
$$

the remainder satisfies $R_{N} \in C^{(N, 1)}([-T, T] \times M)$ and $R_{N}(t, \cdot)$ vanishes at $t=0$, see [10, Sect. 6.6, eq. 6.35]. Moreover, $R_{N}$ is supported on $\{(x, t): \operatorname{dist}(x, S) \leq|t|\}$, all of this implies that, as $t \rightarrow 0^{+}$,

$$
h(t)=\int_{M \backslash S} u(t, x) \mathrm{d} V(x)=\int_{M \backslash S} \tilde{u}^{+}(t, x) \mathrm{d} V(x)+O\left(t^{\infty}\right)
$$

and moreover $h \in C^{\infty}([0, T])$. The structure of $R_{N}$ implies that $\square \tilde{u}^{+}(t, x)=O\left(t^{\infty}\right)$ on $M \backslash S$, provided that this expression is interpreted in a sufficiently weak sense. Formally, therefore

$$
\begin{aligned}
\partial_{t}^{2} \int_{M \backslash S} \tilde{u}^{+}(\cdot, t) \mathrm{d} V & =\int_{M \backslash S} \Delta \tilde{u}^{+}(\cdot, t) \mathrm{d} V+O\left(t^{\infty}\right) \\
& =-\int_{\partial S} \nabla \tilde{u}^{+}(\cdot, t) \cdot v \mathrm{~d} A+O\left(t^{\infty}\right),
\end{aligned}
$$

where the last step is the divergence theorem. One may verify Eq. (14) rigorously by either doing the above steps in the sense of distributions, or by a (somewhat tedious) manual computation. Combining this with Eq. (13),

$$
h^{\prime \prime}(t)=-\int_{\partial S} \nabla \tilde{u}^{+}(\cdot, t) \cdot v \mathrm{~d} A+O\left(t^{\infty}\right) .
$$

The quantity $h^{(j)}(0)$ may thus be seen to depend $\tilde{u}^{+}(0, \cdot)$ at $\partial S$, which in turn depends on $a_{i}^{ \pm}$at $t=0$. Defining $\mathbf{S}_{i}:=a_{i}^{+}+a_{i}^{-}$and $\mathbf{D}_{i}:=a_{i}^{+}-a_{i}^{-}$for $i=0,1, \ldots$, let $L$ be the (spatial) differential operator defined for $w \in C^{\infty}(M)$ by $L w:=\frac{1}{2} \Delta \varphi w-v \cdot \nabla w$. For $i \in \mathbb{N}_{0}$, the transport equations imply

$$
\begin{aligned}
\partial_{t} \mathbf{S}_{0} & =L \mathbf{D}_{0}, \quad \partial_{t} \mathbf{D}_{0}=L \mathbf{S}_{0}, \\
\partial_{t} \mathbf{S}_{i+1} & =L \mathbf{D}_{i+1}-\frac{1}{2} \square \mathbf{D}_{i}, \quad \partial_{t} \mathbf{D}_{i+1}=L \mathbf{S}_{i+1}-\frac{1}{2} \square S_{i} \quad \text { for } \quad i \geq 0,
\end{aligned}
$$


with initial values satisfying

$$
\begin{aligned}
a_{0}^{+}(0, \cdot) & =\frac{1}{2} \mathbf{S}_{0}(0, \cdot)=\frac{1}{2} f(\cdot), \quad \mathbf{D}_{0}(0, \cdot)=0, \\
a_{i+1}^{+}(0, \cdot) & =\frac{1}{2} \mathbf{D}_{i+1}(0, \cdot)=-\frac{1}{2} \partial_{t} \mathbf{S}_{i}(0, \cdot), \quad \mathbf{S}_{i+1}(0, \cdot)=0 .
\end{aligned}
$$

Lemma 4 For $i, n \in \mathbb{N}_{0}$ it holds that $\partial_{t}^{2 n} \mathbf{D}_{i}(0, \cdot)=0$ (note that as a consequence, also $a_{i+1}(0, \cdot), L \mathbf{D}_{i}(0, \cdot)$, and $\square^{n} \mathbf{D}_{i}(0, \cdot)$ are zero $)$.

Proof We will proceed by induction over $i$ and use the identities Eqs. (16)-(19). For $i=0, \mathbf{D}_{0}(0, \cdot)=0$ is trivially satisfied. Moreover, $\partial_{t}^{2 n} \mathbf{D}_{0}=R^{n} \mathbf{D}_{0}$, which is zero at $t=0$. For $i=1$, observe that $a_{1}^{+}(0, \cdot)=-\frac{1}{2} \partial_{t} \mathbf{S}_{0}(0, \cdot)=-\frac{1}{2} L \mathbf{D}_{0}(0, \cdot)=0$, and thus $\mathbf{D}_{1}(0, \cdot)=0$. Likewise, $\partial_{t}^{2} \mathbf{D}_{1}=\partial_{t}\left(L \mathbf{S}_{1}-\frac{1}{2} \square \mathbf{S}_{0}\right)=L\left(L \mathbf{D}_{1}-\frac{1}{2} \square \mathbf{D}_{0}\right)-\frac{1}{2} \square L \mathbf{D}_{0}$. As the operator $L$ commutes with $\partial_{t}^{2}$, this expression vanishes at $t=0$. Induction over $n$ proves the remainder of the statement for $i=1$. For the general case, we assume the induction hypothesis for $i$ and $i+1$ and start by noting that $\mathbf{D}_{i+2}(0, \cdot)=$ $2 a_{i+2}^{+}(0, \cdot)=-\partial_{t} \mathbf{S}_{i+1}(0, \cdot)=-\left(L \mathbf{D}_{i+1}(0, \cdot)-\frac{1}{2} \square \mathbf{D}_{i}(0, \cdot)\right)=0$. Moreover, $\partial_{t}^{2} \mathbf{D}_{i+2}=\partial_{t}\left(L \mathbf{S}_{i+2}-\frac{1}{2} \square \mathbf{S}_{i+1}\right)=L\left(L \mathbf{D}_{i+2}-\frac{1}{2} \square \mathbf{D}_{i+1}\right)-\frac{1}{2} \square\left(L \mathbf{D}_{i+1}-\frac{1}{2} \square \mathbf{D}_{i}\right)$, which again vanishes at $t=0$; the case $n>1$ may again be proven by induction over $n$.

Corollary 5 For even $j \in \mathbb{N}_{\geq 2}$, the $j$ th derivative of $h$ satisfies

$$
h^{(j)}(0)=-\frac{1}{2} \int_{S} \Delta^{j / 2} f \mathrm{~d} V
$$

Proof Lemma 4 shows that for $i \geq 1, a_{i}^{+}(0, x)=0$. Together with Eq. (15), thus $h^{\prime \prime}(0)=-\int_{\partial S} \nabla a_{0}^{+}(0, \cdot) \cdot v \mathrm{~d} A=-\frac{1}{2} \int_{\partial S} \nabla f \cdot v \mathrm{~d} A$. This is the case $j=2$. More generally, for $j=2 k$ with $k \in \mathbb{N}_{\geq 2}$, we use that (for $x \in \partial S$ ), $\tilde{u}^{+}$satisfies $\partial_{t}^{2} \tilde{u}^{+}(t, x)=$ $\Delta \tilde{u}^{+}(t, x)+O\left(t^{\infty}\right)$. Equation 15 ensures that as $t \rightarrow 0^{+}$,

$$
h^{(2 k)}(t)=\int_{\partial S} \nabla\left(\Delta^{k-1} \tilde{u}^{+}(t, \cdot)\right) \cdot v \mathrm{~d} A+O\left(t^{\infty}\right) .
$$

As for the case $k=1$, it follows that $h^{(2 k)}(0)=-\int_{\partial S} \nabla\left(\Delta^{k-1} a_{0}^{+}\right) \cdot v \mathrm{~d} A$, the divergence theorem yields the claim.

The odd coefficients are trickier, we only compute the case $j=3$. We start with the observation that for $x \in \partial S, \varphi^{+}(t, x)=t$ and therefore

$$
\tilde{u}^{+}(t, x)=\sum_{i=0}^{N} \frac{1}{i !} t^{i} a_{i}^{+}(t, x) \text { for } t \geq 0, x \in \partial S .
$$


Recall that the Lie-derivative acts on functions $w \in C^{\infty}(M)$ by $\mathcal{L}_{v} w=\nabla w \cdot v$. Thus $\mathcal{L}_{\nu} \theta_{i+1}\left(\varphi^{+}(t, x)\right)=-\theta_{i}\left(\varphi^{+}(t, x)\right)$, so for $x \in \partial S$,

$$
\mathcal{L}_{\nu} \tilde{u}^{+}(t, x)=\sum_{i=0}^{N-1} \frac{t^{i}}{i !}\left(\mathcal{L}_{\nu} a_{i}^{+}(t, x)-a_{i+1}(t, x)\right)+O\left(t^{\infty}\right)
$$

Therefore $\partial_{t} \mathcal{L}_{\nu} \tilde{u}^{+}(0, x)=\partial_{t}\left(\mathcal{L}_{v} a_{0}^{+}(0, x)-a_{1}^{+}(t, x)\right)+\left(\mathcal{L}_{\nu} a_{1}^{+}(0, x)-a_{2}^{+}(0, x)\right)$, but the second term is zero as $a_{1}^{+}$and $a_{2}^{+}$vanish at $t=0$ by Lemma 4 . Substituting the transport equations and removing further zero terms leaves $\partial_{t} \mathcal{L}_{\nu} \tilde{u}^{+}(0, x)=$ $\mathcal{L}_{v} L a_{0}^{+}(0, x)+\frac{1}{2} \square a_{0}(0, x)=\frac{1}{2}\left(\mathcal{L}_{v} L f(x)-\frac{1}{2} \Delta f(x)+\frac{1}{2} L^{2} f(x)\right)$. Thus (recall that $\left.L=-\mathcal{L}_{v}+\frac{1}{2} \Delta \varphi\right)$ directly from Eq. (15),

$$
h^{(3)}(0)=-\frac{1}{2} \int_{\partial S} \mathcal{L}_{v} L f(x)-\frac{1}{2} \Delta f(x)+\frac{1}{2} L^{2} f(x) \mathrm{d} A(x) .
$$

The formula

$$
\Omega_{S, f}(t)=\int_{0}^{\infty} \hat{k}(s)\left(\int_{S} f \mathrm{~d} V-h(s \sqrt{t})\right) \mathrm{d} s
$$

established in the previous section, together with Lemma 3, yields the asymptotic behaviour of $\Omega_{S, f}(t)$ by taking the Taylor expansion of $h$ using Corollary 5. This gives the remainder of the claims of theorem 2 .

\section{Discussion}

The above-said is not specific to the heat equation. Taking $k(x)=\exp \left(-x^{2 m}\right), m \in \mathbb{N}$, we may, for example, study the one-parameter operator family $\exp \left(-t^{m} \Delta^{m}\right)$. The wave equation estimates needed are the same. For $m \geq 2$, a brief calculation yields the explicit $t \rightarrow 0^{+}$asymptotics

$$
\left\langle\exp \left(t^{m} \Delta^{m}\right) f \mathbb{1}_{S}, \mathbb{1}_{S}\right\rangle=\int_{S} f \mathrm{~d} V-\left(\pi^{-1} \Gamma\left(\frac{2 m-1}{2 m}\right) \int_{\partial S} f \mathrm{~d} A\right) \sqrt{t}+o(t) .
$$

We conclude with the observation that the generalization of this paper to weighted Riemannian manifolds (cf. [4]) is straightforward.

Acknowledgements Open Access funding provided by Projekt DEAL. The author was supported by the Priority Programme SPP 1881 Turbulent Superstructures of the Deutsche Forschungsgemeinschaft. The author thanks the reviewer for simplifying a significant part of the argument, and thanks Oliver Junge and Daniel Karrasch for helping to improve the manuscript.

Open Access This article is licensed under a Creative Commons Attribution 4.0 International License, which permits use, sharing, adaptation, distribution and reproduction in any medium or format, as long as you give appropriate credit to the original author(s) and the source, provide a link to the Creative Commons licence, and indicate if changes were made. The images or other third party material in this article are included 
in the article's Creative Commons licence, unless indicated otherwise in a credit line to the material. If material is not included in the article's Creative Commons licence and your intended use is not permitted by statutory regulation or exceeds the permitted use, you will need to obtain permission directly from the copyright holder. To view a copy of this licence, visit http://creativecommons.org/licenses/by/4.0/.

\section{References}

1. Cheeger, J., Gromov, M., Taylor, M.: Finite propagation speed, kernel estimates for functions of the Laplace operator, and the geometry of complete Riemannian manifolds. J. Differ. Geom. 17(1), 15-53 (1982)

2. Chernoff, P.R.: Essential self-adjointness of powers of generators of hyperbolic equations. J. Funct. Anal. 12(4), 401-414 (1973)

3. Gray, A.: Tubes. Progress in Mathematics, vol. 221, 2nd edn. Springer, Basel (2004)

4. Grigor'yan, A.: Heat Kernel and Analysis on Manifolds. No. 47 in Studies in Advanced Mathematics. AMS, Rhode Island (2009)

5. Lang, S.: Differential and Riemannian Manifolds. Graduate Texts in Mathematics, vol. 160. Springer, New York (1995)

6. Marola, N., Miranda, M., Shanmugalingam, N.: Characterizations of sets of finite perimeter using heat kernels in metric spaces. Potential Anal. 45(4), 609-633 (2016)

7. Miranda Jr., M., D. Pallara, F. Paronetto, Preunkert, M.: Short-time heat flow and functions of bounded variation in $R^{N}$. Ann. Fac. Sci. Toulouse Math. Ser. 6 16(1), 125-145 (2007)

8. Petersen, P.: Riemannian Geometry. Graduate Texts in Mathematics, vol. 171 3rd edn. Springer, New York (2016)

9. Rudin, W.: Functional Analysis. International Series in Pure and Applied Mathematics, 2nd edn. McGraw-Hill Inc, New York (1991)

10. Taylor, M.E.: Partial Differential Equations I. Applied Mathematical Sciences, vol. 115, 2nd edn. Springer, New York (2011)

11. van den Berg, M.: Heat content in non-compact Riemannian manifolds. Integr. Equ. Oper. Theory 90(1), 8 (2018)

12. van den Berg, M., Gilkey, P.: Heat flow out of a compact manifold. J. Geom. Anal. 25, 1576-1601 (2015)

13. van den Berg, M., Gilkey, P.: Heat content asymptotics of a Riemannian manifold with boundary. J. Funct. Anal. 120(1), 48-71 (1994)

Publisher's Note Springer Nature remains neutral with regard to jurisdictional claims in published maps and institutional affiliations. 